\title{
Algorithm for the calculation of vibration inherent frequencies bending from two-shafts transmission
}

\author{
Jan-Cristian Grigore ${ }^{1 *}$, Ion Bulac ${ }^{2}$ \\ ${ }^{1}$ University of Piteşti, jan_grigore@yahoo.com, Romania \\ 2University of Pitești, ionbulac57@yahoo.com, Romania
}

\begin{abstract}
The operation of the speed shaft transmissions at or near the natural frequency of the pulses at the resonance phenomenon leads to bending, when the amplitude of the oscillations increases sharply, causing deterioration or complete destruction thereof. To avoid system resonance operation is necessary to know the most accurate values its pulsations and taking appropriate constructive measures to avoid overlapping with disturbing frequency harmonics (operating speeds).This paper presents an algorithm for calculating the pulsation and vibration modes in bending, and based on numerical simulations performed on a real two-shafts transmission and will draw conclusions drawn diagrams.
\end{abstract}

\section{Introduction}

The frequency of variation of the outer disturbing forces are commonly known and are in most cases due to the operating condition. To establish whether resonance is likely to be determined as accurately self-oscillation frequencies of the system. Disruptive forces but do not have a single frequency spectrum from ), Fl. Dudita [1], Fl. Dudita, D. Diaconescu, Cr. Bohn, M. Neagoe, and R. Saulescu [2], the multi-speed transmission, $n, 2 n, 3 n$ ( $n$ being speed transmission). If we take into account that the angular gear also has several frequencies of oscillation own and operating speeds range between certain limits it will be clear that when working with multiple operating modes can not avoid certain harmonic disturbing forces coincide their oscillation frequencies of transmission. In this case, the task is calculation to determine the oscillation amplitudes and values of stresses occurring in the components of the transmission at resonance.

\section{Constructive model and equivalent mechanical model}

The constructive solution for mobile two-shafts transmission (see Fig. 1.a.), is associated equivalent mechanical model (see Fig. 1.b.), the sections A and D elastic supports are located elastic constants $k_{A}, k_{D}$.

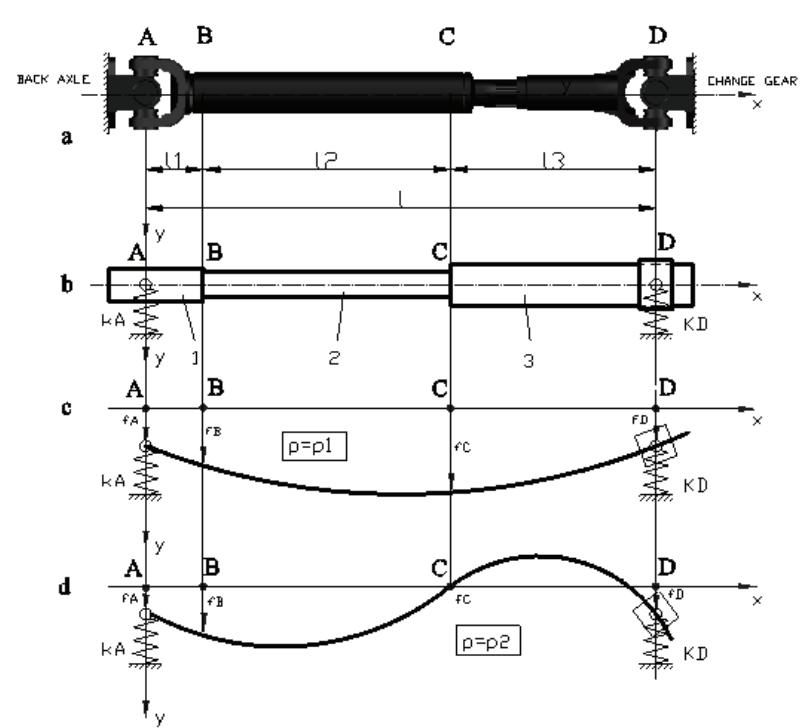

Fig. 1. The construction and mechanical equivalent model

The following, using the notation $f_{i}, \theta_{i}, M_{i}, F_{i}$ respectively arrow, rotation, bending moment and shear force in a current section, R. Voinea, D. Voiculescu and FL. Simion [3], A. Ripianu and I. Craciun [4], N. Pandrea, S. Parlac and D. Popa [5].

1) $\Delta_{i} ; \Delta_{A} ; \Delta_{B} ; \Delta_{C} ; \Delta_{D}$ state vectors defined by the equality:

\footnotetext{
*Corresponding author: jan_grigore@yahoo.com
} 


$$
\begin{aligned}
& \Delta_{i}=\left(\mathbf{f}_{i}, \theta_{i}, M_{i}, F_{i}\right)^{T} \\
& \Delta_{A}=\left(\mathbf{f}_{A}, \theta_{A}, M_{A}, F_{A}\right)^{T} \\
& \Delta_{B}=\left(f_{B}, \theta_{B}, M_{B}, F_{B}\right)^{T} \\
& \Delta_{C}=\left(f_{C}, \theta_{C}, M_{C}, F_{C}\right)^{T} \\
& \Delta_{D}=\left(f_{D}, \theta_{D}, M_{D}, F_{D}\right)^{T} .
\end{aligned}
$$

2) $l_{i}, \rho_{i}, A_{i}, E_{i}, I_{y i}$, respectively, the length, density, area, transverse modulus of elasticity and geometrical moment of inertia mainly for the section corresponding to the index $i=1,2,3$

3) Parameter $\alpha_{\mathrm{i}}$ defined by the relationship:

$$
\alpha_{i}=\sqrt[4]{p^{2} \frac{\rho_{i} A_{i}}{E_{i} \mathbf{I y i}}}
$$

where $p$ is the vibration inherent pulse.

4) Parameter $z_{i}$ defined by the relationship:

$$
\mathbf{z}_{\mathbf{i}}=\alpha_{\mathbf{i}} \cdot \mathbf{l}_{\mathbf{i}} \cdot
$$

5) $\operatorname{chz}, \operatorname{sh} z-\sin$ and cos hyperbolic functions:

$$
\operatorname{ch}\left(\mathbf{z}_{\mathbf{i}}\right)=\frac{\mathrm{e}^{\mathbf{z}_{\mathbf{i}}}+\mathrm{e}^{-\mathbf{z}_{\mathbf{i}}}}{2} ; \operatorname{sh}\left(\mathbf{z}_{\mathbf{i}}\right)=\frac{\mathrm{e}^{\mathbf{z}_{\mathbf{i}}}-\mathrm{e}^{-\mathbf{z}_{\mathbf{i}}}}{2} .
$$

6) $f_{j}\left(z_{i}\right), j=1,2,3,4$, Krâlov functions defined by relations:

$$
\begin{aligned}
& f_{1}\left(z_{i}\right)=\frac{\operatorname{ch}\left(z_{i}\right)+\cos \left(z_{i}\right)}{2} \\
& f_{2}\left(z_{i}\right)=\frac{\operatorname{sh}\left(z_{i}\right)+\sin \left(z_{i}\right)}{2} \\
& f_{3}\left(z_{i}\right)=\frac{\operatorname{ch}\left(z_{i}\right)-\cos \left(z_{i}\right)}{2} \\
& f_{4}\left(z_{i}\right)=\frac{\operatorname{sh}\left(z_{i}\right)-\sin \left(z_{i}\right)}{2} .
\end{aligned}
$$

7) $F(z)-$ Krâlov matrixes defined by relations:

$$
F(z)=\left(\begin{array}{llll}
f_{1}(z) & f_{2}(z) & f_{3}(z) & f_{4}(z) \\
f_{4}(z) & f_{1}(z) & f_{2}(z) & f_{3}(z) \\
f_{3}(z) & f_{4}(z) & f_{1}(z) & f_{2}(z) \\
f_{2}(z) & f_{3}(z) & f_{4}(z) & f_{1}(z)
\end{array}\right)
$$

8) $\alpha, \alpha^{-1}$-for diagonal matrixes:

$$
\alpha=\left(\begin{array}{cccc}
1 & 0 & 0 & 0 \\
0 & \frac{1}{\alpha} & 0 & 0 \\
0 & 0 & -\frac{1}{\alpha^{2} E I_{w}} & 0 \\
0 & 0 & 0 & -\frac{1}{\alpha^{3} E I_{w}}
\end{array}\right)
$$

$$
\alpha^{-1}=\left(\begin{array}{cccc}
1 & 0 & 0 & 0 \\
0 & \alpha & 0 & 0 \\
0 & 0 & -\alpha^{2} \mathbf{E} \mathbf{I}_{w} & 0 \\
0 & 0 & 0 & -\alpha^{3} \mathbf{E} \mathbf{I}_{w}
\end{array}\right) .
$$

9) $R_{i}, i-1,2,3$, field matrixes of sections, I. Bulac [6] :

$$
\mathbf{R}_{\mathbf{i}}=\boldsymbol{\alpha}_{\mathbf{i}}^{-1} \cdot \mathbf{F}\left(\boldsymbol{\alpha}_{\mathbf{i}} \mathbf{x}_{\mathbf{i}}\right) \cdot \boldsymbol{\alpha}_{\mathbf{i}} .
$$

10) $R$, field matrix for all two-shafts transmission limited points $A, D$ :

$$
\mathbf{R}=\mathbf{R}_{\mathbf{3}} \cdot \mathbf{R}_{\mathbf{2}} \cdot \mathbf{R}_{\mathbf{1}} .
$$

From boundary conditions resulting from moments A and D:

$$
\mathbf{M}_{\mathbf{A}}=\mathbf{M}_{\mathbf{D}}=0 .
$$

and shear forces calculated with relations:

$$
\mathbf{F}_{\mathrm{A}}=\mathbf{k}_{\mathrm{A}} \mathbf{f}_{\mathrm{A}} ; \mathbf{F}_{\mathbf{D}}=-\mathbf{k}_{\mathbf{D}} \mathbf{f}_{\mathbf{D}} \text {. }
$$

Therefore, taking into account relations (1) și (11), state vectors of the sections $\mathrm{A}$ and $\mathrm{D}$ are:

$$
\begin{aligned}
& \Delta_{\mathrm{A}}=\left(\frac{\mathbf{F}_{\mathrm{A}}}{\mathbf{k}_{\mathrm{A}}}, \theta_{\mathrm{A}}, \mathbf{0}, \mathbf{F}_{\mathrm{A}}\right)^{\mathrm{T}} \\
& \Delta_{\mathrm{D}}=\left(\mathbf{f}_{\mathrm{D}}, \theta_{\mathrm{D}}, \mathbf{0},-\mathbf{k}_{\mathrm{D}} \mathbf{f}_{\mathrm{D}}\right)^{\mathrm{T}} .
\end{aligned}
$$

Using notations:

$$
\begin{aligned}
\mathbf{T}_{\mathrm{A}} & =\left(\begin{array}{cc}
0 & \frac{1}{\mathbf{k}_{\mathrm{A}}} \\
1 & 0 \\
0 & 0 \\
0 & 1
\end{array}\right) \\
\mathbf{R}^{*} & =\left(\begin{array}{ll}
\mathbf{R}_{11} & \mathbf{R}_{12} \\
\mathbf{R}_{21} & \mathbf{R}_{22} \\
\mathbf{R}_{31} & \mathbf{R}_{32} \\
\mathbf{R}_{41} & \mathbf{R}_{42}
\end{array}\right)=\mathbf{R}_{3} \cdot \mathbf{R}_{2} \cdot \mathbf{R}_{1} \cdot \mathbf{T}_{\mathrm{A}} .
\end{aligned}
$$

state vectors of the points A și D can be written as:

$$
\Delta_{\mathrm{A}}=\mathbf{T}_{\mathrm{A}} \cdot\left(\begin{array}{l}
\theta_{\mathrm{A}} \\
\mathbf{F}_{\mathrm{A}}
\end{array}\right) ; \Delta_{\mathbf{D}}=\mathbf{R}_{\mathbf{3}} \cdot \mathbf{R}_{\mathbf{2}} \cdot \mathbf{R}_{1} \cdot \Delta_{\mathrm{A}} \cdot(
$$

and the second equality in equation (14) becomes:

$$
\left(\begin{array}{c}
\mathbf{f}_{\mathbf{D}} \\
\theta_{\mathrm{D}} \\
\mathbf{0} \\
-\mathbf{k}_{\mathrm{D}} \mathbf{f}_{\mathrm{D}}
\end{array}\right)=\mathbf{R}^{*} \cdot\left(\begin{array}{c}
\boldsymbol{\theta}_{\mathrm{A}} \\
\mathbf{F}_{\mathrm{A}}
\end{array}\right) .
$$

and hence the homogeneous system of equations is obtained in $\theta_{A}, F_{A}, \theta_{D}, f_{D}$ : 


$$
\left\{\begin{array}{c}
\mathbf{f}_{\mathrm{D}}=\mathbf{R}_{11} \theta_{\mathrm{A}}+\mathbf{R}_{12} \mathbf{F}_{\mathrm{A}} \\
\boldsymbol{\theta}_{\mathrm{D}}=\mathbf{R}_{21} \theta_{\mathrm{D}}+\mathbf{R}_{22} \mathbf{F}_{\mathrm{A}} \\
\mathbf{0}=\mathbf{R}_{31} \theta_{\mathrm{A}}+\mathbf{R}_{32} \mathbf{F}_{\mathrm{A}} \\
-\mathbf{k}_{\mathrm{D}} \mathbf{f}_{\mathrm{D}}=\mathbf{R}_{41} \boldsymbol{\theta}_{\mathrm{A}}+\mathbf{R}_{42} \mathbf{F}_{\mathrm{A}}
\end{array} .\right.
$$

For the system (16) to be non-trivial solution, D. Stanescu [7], must be as determinant system is zero:

$$
\Psi(\mathbf{p})=0 \text {. }
$$

where:

$$
\Psi(\mathbf{p})=\left|\begin{array}{rrr}
\mathbf{R}_{11} & \mathbf{R}_{12} & 1 \\
\mathbf{R}_{21} & \mathbf{R}_{22} & 1 \\
\mathbf{R}_{31} & \mathbf{R}_{32} & 0 \\
\mathbf{R}_{41} & \mathbf{R}_{42} & -\mathbf{k}_{\mathbf{D}}
\end{array}\right| .
$$

By solving the characteristic equation (17) set their pulses $p_{1}, p_{2}, \ldots$, . To represent vibration inherent modes, assign arrow $f_{A}$ equal to the numerical value when the system unit (16) results:

$$
\begin{aligned}
& \mathbf{F}_{A}=\mathbf{k}_{A} ; \theta_{A}=-\frac{\mathbf{R}_{32}}{\mathbf{R}_{31}} \mathbf{k}_{A} \\
& \mathbf{f}_{D}=\mathbf{k}_{A}\left(\mathbf{R}_{12}-\mathbf{R}_{11} \frac{\mathbf{R}_{32}}{\mathbf{R}_{31}}\right) \\
& \theta_{D}=\mathbf{k}_{A}\left(\mathbf{R}_{22}-\mathbf{R}_{21} \frac{\mathbf{R}_{32}}{\mathbf{R}_{31}}\right) .
\end{aligned}
$$

Arrows $f_{B}$ and $f_{C}$ from sections $\mathrm{B}$ and $\mathrm{C}$ are the first elements of the matrixes $\Delta_{B}, \Delta_{C}$, data relationships:

$$
\Delta_{\mathrm{B}}=\mathbf{R}_{1} \cdot \mathbf{T}_{\mathrm{A}} \cdot\left(\begin{array}{l}
\boldsymbol{\theta}_{\mathrm{A}} \\
\mathbf{F}_{\mathrm{A}}
\end{array}\right) ; \Delta_{\mathrm{C}}=\mathbf{R}_{2} \cdot \mathbf{R}_{1} \cdot \mathbf{T}_{\mathrm{A}} \cdot\left(\begin{array}{l}
\boldsymbol{\theta}_{\mathrm{A}} \\
\mathbf{F}_{\mathrm{A}}
\end{array}\right)
$$

\section{The calculation algorithm}

For the numerical calculation one uses the following algorithm:

1) The following parameters are considered as known:

$k_{A}, k_{D}, A_{i}, \rho_{i}, E_{i}, I_{i}, l_{i} \quad i=1,2,3$;

2) $A$ value is chosen for the pulsation $p$ and $a$ variation step $\Delta p$;

3) Calculate parameters $\alpha_{i}, i=1,2,3$ with relation (2) and matrixes $\quad \alpha, \alpha^{-1}$ with relations (7);

4) The parameters $z_{i}$ and hyperbolic functions chz $z_{i}, s h z_{i}, i=1,2,3$ using relations (4) and (5);

5) Calculate Krâlov functions $f_{j}\left(z_{i}\right), j=1,2,3,4 ; i=1,2,3 \quad$, and Krâlov matrixes $F(z)$, with relations (5) and (6);

6) Matrixes calculus $R_{i} ; T_{A} ; R^{*}, \quad i=1,2,3$ with relations (8) and (13);

7) Functions calculus $\Psi(p)$ with relation (17);

8) $p$ is replaced with $p+\Delta p$ and the calculations are made again, up when the characteristic equation (17) is satisfied, obtaining inherent pulsations, so being obtained the own pulsations $p_{1}, p_{2}, \ldots$;

9) Is considered $f_{A}=1$ and calculate $F_{A} ; \theta_{A} ; f_{D}$ with relations (19);

10) Is calculated state vectors $\Delta_{B} ; \Delta_{C}$ with relations (20) and extracted arrow $f_{B} ; f_{C}$;

11) Plot graphs vibration modes for the first two inherent pulses.

\section{Numerical application}

It is considered that two-shafts transmission equips an SUV automobile constructive design of which is shown in Fig. 1., for the known:

$k_{A}=k_{D}=85 \cdot 10^{6}(\mathrm{~N} / \mathrm{m}) ; l_{2}=0,1(\mathrm{~m}) ; l_{2}=1,0(\mathrm{~m}) ;$

$l_{3}=0,4(m) ; A_{1}=19,6 \cdot 10^{-4}\left(\mathrm{~m}^{2}\right) ; A_{2}=3,6 \cdot 10^{-4}\left(\mathrm{~m}^{2}\right)$;

$A_{3}=7,1 \cdot 10^{-4}\left(\mathrm{~m}^{2}\right) ; \rho_{1}=\rho_{2}=\rho_{3}=7800\left(\mathrm{~kg} / \mathrm{m}^{3}\right)$.

In a first approximation be considered shaft of the transmission, consisting of three equal segments of a constant solid section resiliently supported at the ends.

Based on the presented algorithm and a computer program developed in Excel or obtained first and second pulsation own values $\mathrm{p}_{1}=283,2368\left(\mathrm{~s}^{-1}\right)$ and $\mathrm{p}_{2}=1117,1629\left(\mathrm{~s}^{-1}\right)$.

Corresponding to this pulse graphs were drawn at the bending vibration inherent modes shown in Fig. 2. and Fig. 3.

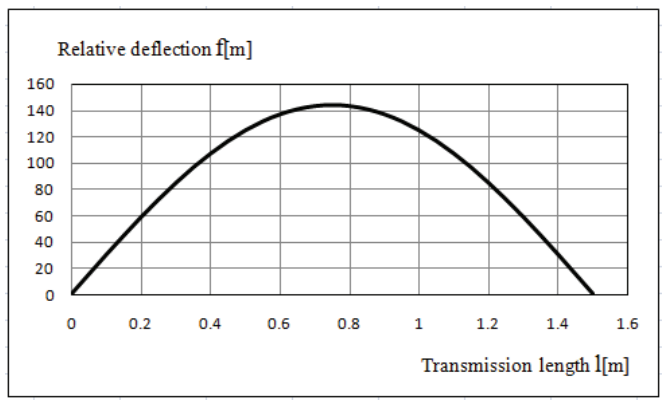

Fig. 2. Vibration mode corresponding to the first inherent pulse for shafts transmission equated with full bar of constant section

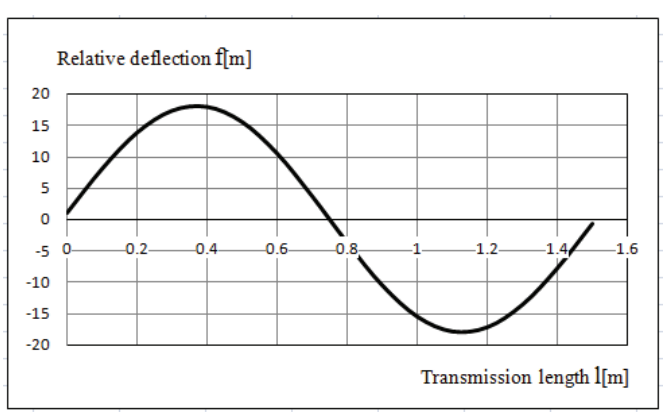

Fig. 3. Vibration mode corresponding to the second inherent pulse for shafts transmission equated with full bar of constant section 
If real two-shafts transmission which is pipe ends with forks bond were obtained first and second inherent pulsation values $\mathrm{p}_{1}=325,2445\left(\mathrm{~s}^{-1}\right), \mathrm{p}_{2}=1228,6995\left(\mathrm{~s}^{-1}\right)$ and vibration inherent modes shown in Fig. 4. and Fig. 5.

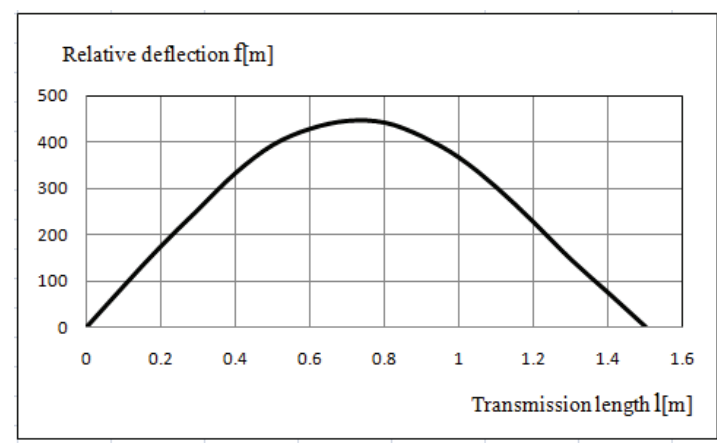

Fig. 4. Vibration mode corresponding to the first inherent pulse for real two-shafts transmission

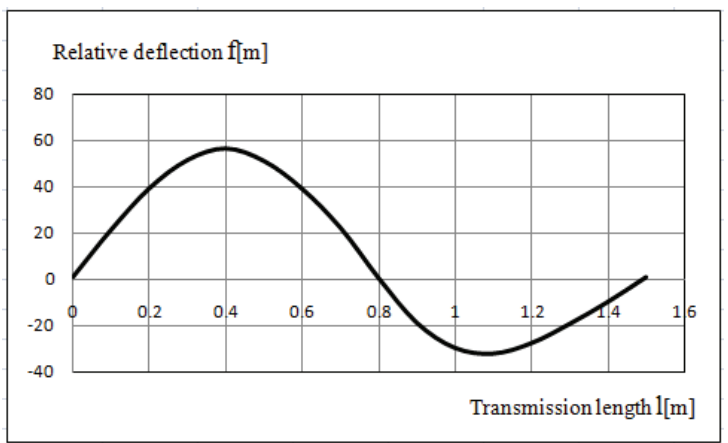

Fig. 5. Vibration mode corresponding to the second inherent pulse for real two-shafts transmission

Taking into account the results and the diagrams of variation of the relative amplitudes along the cardan joint shown in Fig. 2., Fig. 3., Fig. 4. and Fig. 5., there is the following:

1) Two-shaft transmission values for the inherent pulsation its fitting with the shaft are higher than in the case of full drive shaft two-shaft transmission.

2) The positions of the maximum amplitudes occur, for two different cases considered very least, being about the middle of the shaft transmission.
3) For the second inherent pulse give its own shaft section for full maximum amplitudes are equal and opposite and occur at the same distance from the ends at $0,38(\mathrm{~m})$ respectively $11,3(\mathrm{~m})$ to point A (link shaft with rear axle of the car).

4) The actual two-shafts transmission of the maximum amplitudes moves to point $D$ (connection with universal joints reducer gearbox or gearbox), at distances $0,45(\mathrm{~m})$ respectively $1,2(\mathrm{~m})$ to the same reference point $A$, being of different sizes.

\section{Conclusions}

The algorithm presented in this paper and the computer program developed allows numerical study of the influence of geometrical and mechanical parameters of the two-shaft transmission constructive on inherent pulsation and vibration modes in bending.

Numerical simulations can provide important information on of the behavior of the transmission at different operating conditions and taking appropriate constructive measures to avoid negative effects of the phenomenon of resonance.

\section{References}

[1] Fl. Dudita, Cardan shafting, Technical Publishing House, Bucharest, 1966.

[2] Fl. Dudita, D. Diaconescu, Cr. Bohn, M. Neagoe, R. Saulescu, Cardan shafting, Transilvania Expres Publishing House, Brasov, 2003.

[3] R. Voinea, D. Voiculescu, FL. Simion, Introduction in the solid mechanics with applications in engineering, R.S.R. Academy, 1989.

[4] A. Ripianu, I. Craciun, Axles, righteous shafts and crankshafts, Technical Publishing House, Bucharest, 1977.

[5] N. Pandrea, S. Parlac, D. Popa, Models for studying automotive vibrations, Tiparg Publishing House, Pitești, 2001.

[6] I. Bulac, Vibrations of the bicardanic transmissions with elastic supports, 5th Symposium „Durability and Reliability of Mechanical Systems", SYMECH 2012, Târgu-Jiu, Romania, 1819 May, 2012.

[7] D. Stanescu, Numerical methods, Didactic and Pedagogical Publishing House, Bucharest, 2007.

\footnotetext{
*Corresponding author: jan_grigore@yahoo.com
} 